viding another possible mechanism for the effectiveness of chronic ACE inhibition. Also, although the current study focuses primarily on the effect of chymase release on Ang II levels, chymase contributions to adverse LV remodeling may possibly be more important. If Ang II is not actually the main effector molecule, a better understanding of how ACEIs exert their effects could lead to further improvements in therapeutic outcomes.

\section{Acknowledgments}

This work was supported in part by NIH grants R01 HL67724, R01 AG023039, R01 HL091469, P01 HL69020, and P01 AG027211 and the Foundation Leducq Transatlantic Network of Excellence.

Address correspondence to: Junichi Sadoshima, Cardiovascular Research Institute, UMDNJ - Newark, 185 S. Orange Ave., MSB G609, Newark, New Jersey 07103. Phone: 973.972.8619; Fax: 973.972.8919; E-mail: sadoshju@umdnj.edu.

1. Nicholls MG, Robertson JI, Inagami T. The reninangiotensin system in the twenty-first century. Blood Press. 2001;10(5-6):327-343.

2. Kurdi M, De Mello WC, Booz GW. Working outside the system: an update on the unconventional behavior of the renin-angiotensin system components. Int J Biochem Cell Biol. 2005;37(7):1357-1367.

3. Ferrario CM. New physiological concepts of the renin-angiotensin system from the investigation of precursors and products of angiotensin I metabolism. Hypertension. 2010;55(2):445-452.

4. Wei CC, et al. Angiotensin peptides modulate bradykinin levels in the interstitium of the dog heart in vivo. J Pharmacol Exp Ther. 2002;300(1):324-329.

5. Kumar R, Singh VP, Baker KM. The intracellular renin-angiotensin system: implications in cardiovascular remodeling. Curr Opin Nephrol Hypertens. 2008; 17(2):168-173.

6. Crackower MA, et al. Angiotensin-converting enzyme 2 is an essential regulator of heart function. Nature. 2002;417(6891):822-828.

7. Dell'Italia LJ, Husain A. Dissecting the role of chymase in angiotensin II formation and heart and blood vessel diseases. Curr Opin Cardiol. 2002; 17(4):374-379.

8. Kaplan AP, Joseph K, Silverberg M. Pathways for bradykinin formation and inflammatory disease. J Allergy Clin Immunol. 2002;109(2):195-209.

9. Bader M, Ganten D. Update on tissue renin-angiotensin systems. J Mol Med. 2008;86(6):615-621.

10. Dickstein K, et al. ESC guidelines for the diagnosis and treatment of acute and chronic heart failure 2008: the Task Force for the diagnosis and treatment of acute and chronic heart failure 2008 of the European Society of Cardiology. Developed in collaboration with the Heart Failure Association of the ESC (HFA) and endorsed by the European Society of Intensive Care Medicine (ESICM). EurJ Heart Fail. 2008;10(10):933-989.

11. Biollaz J, Brunner HR, Gavras I, Waeber B, Gavras H. Antihypertensive therapy with MK 421: angiotensin II--renin relationships to evaluate efficacy of converting enzyme blockade. J Cardiovasc Pharmacol. 1982;4(6):966-972.
12. Wei C-C, et al. Mast cell chymase limits the cardiac efficacy of Ang I-converting enzyme inhibitor therapy in rodents. J Clin Invest. 2010;120(4):1229-1239.

13. Dell'Italia LJ, et al. Compartmentalization of angiotensin II generation in the dog heart. Evidence for independent mechanisms in intravascular and interstitial spaces. J Clin Invest. 1997;100(2):253-258.

14. Wei CC, et al. Evidence for angiotensin-converting enzyme- and chymase-mediated angiotensin II formation in the interstitial fluid space of the dog heart in vivo. Circulation. 1999;99(19):2583-2589.

15. Urata H, Kinoshita A, Misono KS, Bumpus FM, Husain A. Identification of a highly specific chymase as the major angiotensin II-forming enzyme in the human heart. J Biol Chem. 1990; 265(36):22348-22357.

16. The CONSENSUS Trial Study Group. Effects of enalapril on mortality in severe congestive heart failure. Results of the Cooperative North Scandinavian Enalapril Survival Study (CONSENSUS). NEngl J Med. 1987;316(23):1429-1435.

17. The SOLVD Investigators. Effect of enalapril on survival in patients with reduced left ventricular ejection fractions and congestive heart failure. N Engl J Med. 1991;325(5):293-302.

18. Krum H, et al. Effect of valsartan added to background ACE inhibitor therapy in patients with heart failure: results from Val-HeFT. Eur J Heart Fail. 2004;6(7):937-945.

19. McMurray JJ, et al. Effects of candesartan in patients with chronic heart failure and reduced left-ventricular systolic function taking angiotensin-convertingenzyme inhibitors: the CHARM-Added trial. Lancet. 2003;362(9386):767-771.

20. Mann JF, et al. Renal outcomes with telmisartan, ramipril, or both, in people at high vascular risk (the ONTARGET study): a multicentre, randomised, double-blind, controlled trial. Lancet. 2008; 372(9638):547-553

\title{
Epithelial-mesenchymal transitions and hepatocarcinogenesis
}

\author{
Janice Jou and Anna Mae Diehl
}

Division of Gastroenterology, Duke University, Durham, North Carolina.

\begin{abstract}
Epithelial-mesenchymal transitions (EMTs) are believed to play a role in invasion and metastasis of many types of tumors. In this issue of the JCI, Chen et al. show that a gene that has been associated with aggressive biology in hepatocellular carcinomas initiates a molecular cascade that results in EMT.
\end{abstract}

Hepatocellular carcinoma (HCC) is the fifth most commonly diagnosed, and the third most deadly, cancer worldwide (1). In the United States between 1975 and 2006, HCC was the only cancer with increasing mortality in men and women (2). Surveillance in patients at increased risk for developing HCC has been recommended for

Conflict of interest: The authors have declared that no conflict of interest exists.

Citation for this article: J Clin Invest. 2010; 120(4):1031-1034. doi:10.1172/JCI42615. detection of early HCC (3); however, this is underutilized, and many patients present with locally advanced or metastatic disease. These patients are not liver transplantation candidates and have limited therapeutic options. Thus, there is an urgent need for identification of patients at risk for HCC and further risk stratification of these patients to improve the outcomes. Therefore, groups like Chen et al. (4) are applying gene profiling to identify better prognostic and/or therapeutic targets. Their present work, in this issue of the JCI, reveals that the oncogene chromodomain helicase/ATPase DNA binding protein 1-like gene $(C D H 1 L)$ is commonly amplified in HCC (4).

Chen et al. demonstrated why CDH1L amplification is important in HCC (Figure 1 and ref. 4). CDH1L functions as a transcription factor that induces expression of the guanine nucleotide exchange factor (GEF) ARHGEF9. GEFs catalyze exchange of GDP for GTP on RhoGTPases, a family of GTPases that includes Rho and Rac proteins as well as Cdc42 (5). Chen et al. demonstrated that Cdc42 was the target of ARHGEF9 and that increased ARHGEF9 enhanced formation of Cdc42-GTP (i.e., activated Cdc42) in HCC cells (4). Cdc42 collaborates with other Rho kinases to modulate assembly and disassembly of the actin cytoskeleton 


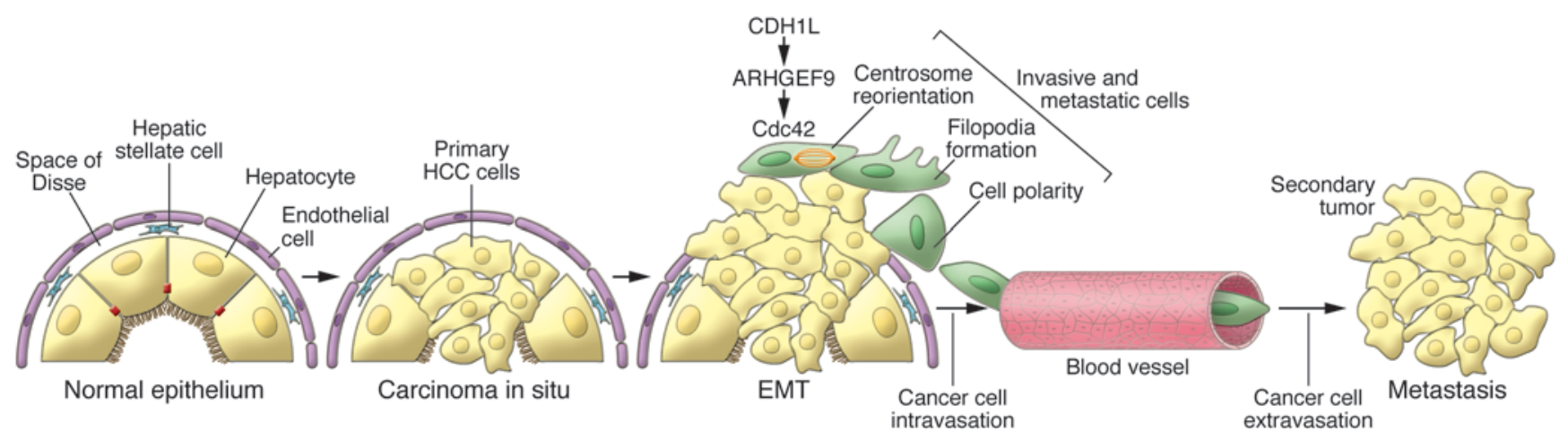

Figure 1

Hepatocarcinogenesis and EMT. In healthy livers, hepatocytes exhibit features of epithelial cells because they are polarized and adherent to each other. However, unlike epithelial cells in other tissues, healthy hepatocytes lack a basement membrane. Hence, healthy, mature hepatocytes are not physically separated from the adjacent stroma and hepatic stellate cells that reside in the space of Disse, sandwiched between the hepatic epithelia and fenestrated endothelial cells that line hepatic sinusoids. Indeed, because the sinusoids themselves also lack a typical basement membrane, endothelial cells, hepatic stellate cells, and hepatocytes all coexist within the same mesenchyme. During hepatocarcinogenesis, genetic events that enhance the migratory capabilities of hepatocytes may occur, and the normal absence of membrane barriers to physically separate hepatocytes from the hepatic sinusoids permits easy entry of motile neoplastic hepatocytes into the vascular system. The current study by Chen et al. (4) delineates a mechanism by which malignant hepatocytes become more motile and invasive. Namely, amplification of CDH1L results in induction of ARHGEF9 and resultant activation of the small GTP-binding protein Cdc42. Cdc42-GTP orchestrates various events, including reorganization of cytoskeletal elements that permit neoplastic hepatocytes to migrate away from neighboring epithelial cells, move through the space of Disse, invade neighboring vascular spaces, and be swept along with the blood flow to other parts of the hepatic lobule and/or out of the liver to extrahepatic sites.

and has been shown to regulate establishment of cell polarity, filopodia formation, and centrosome reorientation in migrating cells. Protein kinase cascades that control cell viability and proliferative activity, including JNK, p38MAPK, and p21-associated protein kinases (PAK), are also downstream targets of Cdc42-GTP (5). Activating $\mathrm{Cdc} 42$ induced HCC cells to undergo changes in the actin cytoskeleton, filopodia formation, adherens junction disruption, and cell migration, which suggests that generating invasive cancer cells in damaged livers involves induction of an epithelialmesenchymal transition (EMT).

\section{EMT and HCC}

EMT is a complex process through which epithelial cells gradually dissolve connections to adjacent cells, rearrange their cytoskeletons, upregulate production of matrix remodeling factors, and migrate out of epithelial sheets into adjacent stroma. EMT occurs routinely during development and contributes to fetal tissue formation. It also occurs during some wound healing responses in adults and is believed to be a major contributing mechanism to cancer invasion and metastasis (6-8). Consistent with this information, the current work demonstrated that $C D H 1 L$-driven expression of AFHGEF9, and resultant activation of Cdc42 and EMT, was asso- ciated with venous invasion, formation of microsatellite tumors and metastases, and reduced survival from HCC in mice and with increased vascular invasion and reduced survival in HCC patients (4). Thus, CDH1L is one of the factors that promote EMT in HCC.

This discovery compliments and extends growing evidence that implicates EMT in hepatocarcinogenesis. For example, tetraspanin TM4SF5-mediated EMT causes loss of contact inhibition and uncontrollable growth of cultured human hepatocarcinoma cells, as well as tumor formation and/or metastasis in mice $(9,10)$. The pro-EMT transcription factors Twist and Snail are expressed in about $40 \%-70 \%$ of HCCs and correlate with evidence of adherens junction disruption and a worse prognosis $(11,12)$. More than $60 \%$ of HCCs express eukaryotic initiation factor $5 \mathrm{~A} 2$ (EIF5A2), with the highest levels occurring in metastatic cancers. EIF5A2 activates Rho kinases to stimulate formation of stress fibers, lamellipodia, EMT, and HCC cell migration (13). The hepatitis B $\mathrm{X}$ gene (HBx; ref. 14) and hepatitis $\mathrm{C}$ core proteins (15) induce EMT in cultured liver cells, consistent with evidence that chronic viral hepatitis increases the risk for HCC. $H B x$ promotes EMT by activating STAT $5 b$, and the level of STAT5b activation in HCC correlates with advanced tumor stage, venous infiltration, and microsatellite formation (14). Hepatoma cell lines also differ somewhat in their endogenous expression of putative EMT regulators, with the greatest expression occurring in lines expressing more mesenchymal proteins, such as Hep3B $(16,17)$. The Hedgehog pathway is active in Hep3B cells and is required for growth (18). Hedgehog signaling promotes EMT during development and in many other types of cancers (19). Moreover, pathway activation is common in HCC and correlates with aggressive tumor biology (20). Elevated expression of another EMT mediator, integrin-linked kinase (ILK), identifies hepatoma cell lines that are resistant to epidermal growth factor receptor-targeted therapies, and knocking down ILK restores sensitivity to these agents (21). EMT is also induced in neoplastic hepatocytes by TGF- $\beta$, IL-like EMT inducer (ILE1), and oncogenic Ras interactions that promote distant metastasis (22). Similarly, ectopic expression of Twist or Snail in hepatoma cell lines with low endogenous expression of these factors enhances cancer cell motility and invasiveness $(12,17)$. Thus, several mechanisms appear capable of stimulating EMT in HCC, and, regardless of the specific factor responsible for EMT induction, HCCs with features of EMT consistently demonstrate more vascular invasion, metastases, 
and a poorer prognosis than do HCCs lacking EMT characteristics.

Evidence demonstrating that EMT correlates with aggressive biology in HCC suggests that EMT conveys certain survival advantages to tumor cells. These include escape from apoptotic stimuli that are prevalent within the primary tumor (TGF- $\beta$, hypoxia) and improved access to nutrients and/or growth factors (by migrating to sites remote from the competing forces that are operative in the primary tumor). Cells that have undergone EMT also acquire the ability to generate factors that stimulate production of vasculature and stroma. This property sustains the outgrowth of new epithelial tumor nodules within or near the primary tumor and after the subpopulations of the metastatic (mesenchymal-appearing) tumor cells have arrived in distant sites and undergone mesenchymal-epithelial transition (MET) to reacquire their epithelial phenotype (7). It is therefore tempting to speculate that situations that promote hepatocarcinogenesis stimulate EMT and that liver cells that have acquired pro-EMT modifications, such as CDH1L amplifications, might have a growth advantage in such environments.

\section{Cirrhosis, EMT, and HCC}

The single greatest risk factor for HCC in humans is cirrhosis (3). Cirrhosis is a conserved response to various types of chronic liver injury that kill liver epithelial cells (i.e., hepatocytes and cholangiocytes). It is the result of suboptimal repair that is unable to fully regenerate or replace dead liver epithelial cells, thereby maintaining stimuli for tissue remodeling that result in chronic expansion of liver epithelial progenitor populations, ongoing inflammation, angiogenesis, and fibrogenesis. Hence, cirrhosis is a consequence of unsuccessful wound healing. In many tissues (e.g., kidney, skin, and lung), chronic wound healing responses that lead to fibrosis provide a stimulus for EMT (6). However, whether EMT occurs during chronic fibrosing liver injury remains controversial $(23,24)$.

As recently reviewed elsewhere (25), there is consistent evidence that profibrogenic factors that increase during cirrhosis, such as TGF- $\beta$, can induce hepatocytes and cholangiocytes to undergo EMT in culture. Populations of cells that typically accumulate in cirrhotic livers, such as hepatic stellate cells and progenitors, also have features of cells that are undergoing EMT. In addition, EMT-inducing mechanisms become acti- vated during cirrhosis, and various markers of EMT have been well documented in liver tissues of rodents and patients with various types of chronic liver disease. However, rigorous lineage-tracing proof that EMT actually occurs in situ during cirrhosis, and which cells are involved, is still lacking. With regard to the latter point, it is important to emphasize that lineage-tracing studies that failed to demonstrate EMT in certain types of liver cells $(26,27)$ do not prove that EMT is impossible in other liver cell types, despite recent speculation that a role for EMT in liver repair has been largely refuted (24). Rather, additional research is needed to resolve the debate about noncancer cell EMT in chronically damaged livers, particularly given the compelling evidence from many groups - including Chen et al. - that malignant liver cells are capable of undergoing EMT in this context.

Indeed, if further research were to prove that EMT occurred more generally during fibrogenic wound healing responses induced by chronic liver injury, this might provide an explanation for the strong association between cirrhosis and primary liver cancer (3). Namely, situations that result in cirrhosis apply chronic selection pressure that confers advantage to the outgrowth of cells maximally successful at undergoing EMT. These may include healthy liver epithelial progenitors as well as progenitors that have acquired chromosomal alterations (such as CDH1L amplifications) that enhance their capacity for EMT. If other growth-dysregulatory mutations develop, the latter cells then become cancer stem/ initiating cells that fuel aggressive biology in HCC. Several lines of historical evidence support the concept that EMT is the biological bridge that links cirrhosis and HCC. First, markers of EMT have been detected in the blood of rodents and humans with cirrhosis $(28,29)$. Second, various events that enhance the efficiency of cancer cell EMT predict cancer invasion, metastasis, and reduced survival in HCC patients. Third, circulating EMT markers are most elevated in HCC patients with metastatic cancer $(29,30)$. The present study by Chen et al. advances this field by demonstrating that CDH1L-ARHGEF9-Cdc42 signaling plays an important role in regulating liver cell EMT (4). Their data suggest that malignant liver cells that acquire the ability to activate this pathway in a cell-autonomous fashion evade mechanisms that normally control EMT, permitting them to repopulate and potentially overgrow damaged livers. This knowledge, in turn, identifies molecular targets that might be exploited to improve HCC screening, allocation of existing HCC therapies, and development of novel HCC treatments for cirrhotic patients.

\section{Acknowledgments}

The authors' work is supported by NIDDK/ NIAAA, NIH, grants RO1 DK077794 and RO1 AA01054.

Address correspondence to: Anna Mae Diehl, Gastroenterology Division, Duke University, Snyderman Building (GSRB1), Suite 1073, LaSalle Street, Durham, NC 27710. Phone: 919.684.2366; Fax: 919.684.4183; E-mail: dieh1004@mc.duke.edu.

1. Parkin DM. Global cancer statistics in the year. 2000. Lancet Oncol. 2001;2(9):533-543.

2. Edwards BK, et al. Annual report to the nation on the status of cancer, 1975-2006, featuring colorectal cancer trends and impact of interventions (risk factors, screeingin, and treatment) to reduce future rates. Cancer. 2010;116(3):544-573.

3. Bruix J, Sherman M. Management of hepatocellular carcinoma. Hepatology. 2005;42(5):1208-1236.

4. Chen L, et al. CHD1L promotes hepatocellular carcinoma progression and metastasis in mice and is associated with these processes in human patients. J Clin Invest. 2010;120(4):1178-1191.

5. Hall A. Rho GTPases and the control of cell behaviour. Biochem Soc Trans. 2005;33(Pt5):891-895.

6. Kalluri R, Weinberg RA. The basics of epithelial-mesenchymal transition. J Clin Invest. 2009; 119(6):1420-1428.

7. Acloque H, Adams MS, Fishwick K, Bronner-Fraser M, Nieto MA. Epithelial-mesenchymal transitions: the importance of changing cell state in development and disease. J Clin Invest. 2009;119(6):1438-1449.

8. Zeisberg M, Neilson EG. Biomarkers for epithelial-mesenchymal transitions. J Clin Invest. 2009; 119(6):1429-1437.

9. Lee SA, et al. Tetraspanin TM4SF5 mediates loss of contact inhibition through epithelial-mesenchymal transition in human hepatocarcinoma. J Clin Invest. 2008;118(4):1354-1366.

10. Lee SA, et al. Blockade of four-transmembrane L6 family member 5 (TM4SF5)-mediated tumorigenicity in hepatocytes by a synthetic chalcone derivative. Hepatology. 2009;49(4):1316-1325.

11. Sun T, et al. Expression and functional significance of Twist 1 in hepatocellular carcinoma: Its role in vasculogenic mimicry. Hepatology. 2009;51(2):545-556.

12. Yang $\mathrm{MH}$, et al. Comprehensive analysis of the indpeendent effect of twist and shanil in promoting metastasis of hepatocellular carcinoma. Hepatology. 2009;50(5):1464-1474.

13. Tang DJ, et al. Overexpression of eukaryotic initiation factor $5 \mathrm{~A} 2$ enhances cell motility and promotes tumor metastasis in hepatocellular carcinoma [published online ahead of print November 30, 2009]. Hepatology. doi:10.1002/hep. 2345.

14. Lee TK, et al. Signal transducers and activators of transcription $5 \mathrm{~b}$ activation enhances hepatocellular carcinoma aggressieness through induction of epithelial-mesenchymal transition. Cancer Res. 2006; 66(20):9948-9956.

15. Battaglia $S$, et al. Liver cacncer-derived hepatitis $C$ virus core proteins shift TGF-beta responses from tumor suppression to epithelial-mesenchymal transition. PLoS One. 2009;4(2):e4355.

16 . Slany A, et al. Cell characterization by proteom pro- 
filing applied to primary hepatoctyes and hepatocyte cell lines Hep-G2 and Hep-3B.J Proteome Res. 2010;9(1):6-21

17. Matsuo N, et al. Twist expression promotes migration and invasion in hepatocellular carcinoma. BMC Cancer. 2009;9:240.

18. Sicklick JK, et al. Dysregulation of the Hedgehog pathway in human hepatocarcinogenesis. Carcinogenesis. 2006;27(4):748-757.

19. Katoh Y, Katoh M. Hedgehog signaling, epithelialto-mesenchymal transition and miRNA (review). Int J Mol Med. 2008;22(3):271-275.

20. Katoh Y, Katoh M. Hedgehog target genes: mechanisms of carcinogenesis induced by aberrant hedgehog signaling activation. Curr Mol Med. 2009;9(7):873-886

21. Fuchs BC, et al. Epithelial-to-mesenchymal transition and integrin-linked kinase mediate sensitivity to epidermal growth factor receptor inhibition in human hepatoma cells. Cancer Res. 2008;68(7):2391-2399.

22. Lahsnig C, et al. ILE1 requires oncogenic Ras for the epithelial to mesecnhymal transition of hepatocytes and liver carcinoma progression. Oncogene. 2009;28(5):638-650.

23. Parola M, Pinzani M. Hepatic wound repair. Fibrogenesis Tissue Repair. 2009;2(1):4.

24. Wells RG. The epithelial-to-mesenchymal transition in liver fibrosis: here today, gone tomorrow? [published online ahead of print January 27, 2010]. Hepatology. doi:10.1002/hep.23529.

25. Choi SS, Diehl AM. Epithelial-to-mesenchymal transitions in the liver. Hepatology. 2009;50(6):2007-2013.

26. Sackett SD, et al. Foxl1 is a marker of bipotential hepatic progenitor cells in mice. Hepatology.
2009;49(3):920-929.

27. Taura K, et al. Hepatocytes do not undergo epithelial-mesenchymal transition in liver fibrosis in mice [published online ahead of print October 13, 2009]. Hepatology. doi:10.1002/hep.23368.

28. Witek RP, et al. Liver cell-derived microparticles activate hedgehog signaling and alter gene expression in hepatic endothelial cells. Gastroenterology. 2009; 136:320-330.

29. Min AL, et al. High expression of Snail mRNA in blood from hepatocellular carcinoma patients with extra-hepatic metastasis. Clin Exp Metastasis. 2009; 26(7):759-767.

30. Murata K, Suzuki H, Okano H, Oyamada T, Yasuda T, Sakamoto A. Hypoxia-induced des-gamma-carboxy prothrombin production in hepatocellular carcinoma. Int J Oncol. 2010;36(1):161-170.

\title{
Monkeying around with cardiac progenitors: hope for the future
}

\author{
Li Qian and Deepak Srivastava \\ Gladstone Institute of Cardiovascular Disease, Department of Pediatrics, and \\ Department of Biochemistry and Biophysics, University of California, San Francisco, California
}

\begin{abstract}
Multipotent cardiovascular progenitor cells derived from ES cells or induced pluripotent stem (iPS) cells are an intriguing source for stem cellbased therapies for congenital and acquired heart diseases. From a clinical perspective, the ideal cardiac progenitor cells are those that can proliferate, survive, and differentiate into multiple mature cardiac cell types when transplanted into normal or diseased heart. In this issue of JCI, Blin et al. report the isolation and characterization of a group of early mesodermal cardiovascular progenitor cells, induced by BMP2 and marked by the cell surface protein, stage-specific embryonic antigen 1 (SSEA-1). BMP2induced SSEA-1 ${ }^{+}$cells were purified from ES and iPS cells and could be directed to differentiate into cardiomyocytes, endothelial cells, and smooth muscle cells by treatment with defined cytokines and signaling molecules. Most importantly, purified SSEA ${ }^{+}$progenitor cells from Rhesus monkey ES cells engrafted into nonhuman primate hearts, in which they differentiated into cardiac cells without forming teratomas. These findings move the field another step closer to clinical use of ES or iPS cell-derived cardiovascular progenitors in cardiac repair.
\end{abstract}

Heart failure is a progressive disease that affects over 5 million individuals in the United States. In addition, many of the over 1 million survivors of congenital heart disease are destined to develop heart failure as they age. Current pharmacologic therapies have limited efficacy and only slow the progression of cardiac dysfunction. Heart transplantation is often the last resort of treatment, but the limited donor pool makes this option unrealistic for the

Conflict of interest: Deepak Srivastava serves on the Scientific Advisory Board of iPierian Inc. and RegeneRx Pharmaceuticals.

Citation for this article: J Clin Invest. 2010; 120(4):1034-1036. doi:10.1172/JCI42643. vast majority of patients. Given the disease burden and the unsatisfying therapeutic modalities for heart failure, the potential of cardiac regenerative medicine has generated tremendous interest worldwide.

Approaches to regenerate functional myocardium in damaged hearts have made substantial strides in recent years. Of major tiple cardiovascular progenitor cells (CPCs) that have embryonic origin and can be isolated from heart tissue, or that can be differentiated from ES or induced pluripotent stem (iPS) cells (1-4). The previously characterized CPCs retain intrinsic competence to differentiate into various cardiac lineages. Among them, $\mathrm{Flk}^{+}\left(\mathrm{KDR}^{+}\right.$in human) preimportance was the identification of mul- cursors retain the capacity to differentiate into blood cells and three of the major cell types of the heart cells, namely cardiomyocytes, smooth muscle cells, and endothelial cells $(5,6)$. CPCs expressing the transcription factor Isl1 are also multipotent and give rise to cardiomyocytes, smooth muscle cells, and endothelial cells (7-9). Finally, CPCs marked by the transcription factor $\mathrm{Nkx} 2.5$ are more lineage restricted but can differentiate into cardiomyocytes and smooth muscle cells (10). Despite these advances, practical isolation of cardiac lineage-committed progenitor cells using a surface marker and introduction of such cells into a damaged heart has remained problematic.

\section{Isolation of cardiovascular progenitors using a cell-surface marker}

In this issue of the JCI, Blin and colleagues identified a very early cardiac progenitor population derived from primate (human and monkey) ES and iPS cells. They used knowledge from embryonic development and mimicked the conditions that may be present in the early primitive streak, just as epiblasts give rise to newly formed mesodermal cells that are destined to acquire a cardiac fate (Figure 1). Such cells express transient but high levels of the transcription factors Oct4 and Mesp1 (11). Blin et al. treated ES and iPS cells with BMP2, which activates Wnt3a, to simulate early epiblast conditions, and then used magnetic 